\title{
Evolução das manifestações pré-linguísticas em crianças normais no primeiro ano de vida
}

\author{
Evolution of pre-linguistic manifestations in normal infants \\ during the first year of life
}

\author{
Fleming Salvador Pedroso ${ }^{1}$, Newra Tellechea Rotta ${ }^{2}$, Marlene Canarim Danesi ${ }^{3}$, \\ Lia Nunes de Avila ${ }^{4}$, Carla Baggio Savio ${ }^{5}$
}

\begin{abstract}
RESUMO
Objetivo: A presente pesquisa teve como objetivo determinar a frequência e a ocorrência de elementos pré-linguísticos em crianças normais durante o primeiro ano de vida. Métodos: A amostra constituiu-se de 33 crianças nascidas a termo e normais, no Hospital de Clínicas de Porto Alegre, selecionadas aleatoriamente para um estudo de coorte. As informações, de acordo com as variáveis sorriso social, sons inarticulados, balbucio monossilábico, balbucio polissilábico, primeiras palavras/palavras-frase, jargão e frases, foram obtidas através do inquérito (informações dos pais), da observação espontânea do examinador e de estímulos facilitadores. Resultados: A partir dos resultados obtidos, é possível estabelecer um padrão de desenvolvimento normal das manifestações prélinguísticas. Conclusões: Os resultados indicaram a necessidade de revisar dados de avaliação de linguagem já estabelecidos por pesquisas realizadas no passado e as avaliações em uso atualmente.
\end{abstract}

Descritores: Desenvolvimento da linguagem; Fonética; Criança

\section{INTRODUÇÃO}

Linguagem é um sistema simbólico usado para representar os significados dentro de uma cultura, sendo sua relação entre o conceito e sua forma de expressão essencialmente arbitrária $^{(1)}$. Na visão de Chomsky ${ }^{(2)}$, resumidamente, a linguagem é inata ao ser humano, o qual está naturalmente provido de um "programa" que lhe permite aprender a linguagem quando exposto a uma língua qualquer.

O fato de que cada criança, em tão pouco tempo (aproximadamente três ou quatro anos), se torna um membro da sua

Trabalho realizado no Hospital de Clínicas de Porto Alegre - Unidade de Neuropediatria, pelo Programa de Pós-graduação em Reabilitação e Inclusão do Centro Universitário Metodista IPA - Porto Alegre (RS), Brasil.

(1) Doutor, Professor do Centro Universitário Metodista IPA - Porto Alegre (RS), Brasil.

(2) Doutora, Professora Adjunto do Departamento de Pediatria da Universidade Federal do Rio Grande do Sul - UFRGS - Porto Alegre (RS), Brasil.

(3) Mestre, Professora do Centro Universitário Metodista IPA - Porto Alegre (RS), Brasil.

(4) Pós-graduanda do Programa de Pós-Graduação em Pediatria da Faculdade de Ciências Médicas da Universidade Federal do Rio Grande do Sul - UFRGS

- Porto Alegre (RS), Brasil.

(5) Mestre, Fonoaudióloga da Associação Brasileira Beneficente de Reabilitação - ABBR - Rio de Janeiro (RJ), Brasil.

Endereço para correspondência: Fleming Salvador Pedroso. R.

Praça das Nações Unidas, 61/306, Porto Alegre - RS, CEP: 90690-230.

E-mail: flep@terra.com.br

Recebido em: 28/1/2008; Aceito em: 29/7/2008 comunidade linguística, sendo capaz de produzir e compreender uma variedade de enunciados na língua em que esteve exposta sem, praticamente, nenhum ensino consciente da parte dos adultos, tem despertado grande interesse entre os estudiosos da aquisição da linguagem ${ }^{(1,3-6)}$. $\mathrm{Na}$ atualidade, tem sido dada uma maior importância às produções pré-linguísticas como indicadores precoces da futura fase linguística ${ }^{(1,4,7-8)}$. O desenvolvimento da linguagem segue uma sequência mais ou menos constante e depende, em parte da programação genética, e também de ouvir a fala das outras pessoas, o que significa estímulo ambiental ou input. O exercício adequado da função cognitiva e as oportunidades para a prática da fala incluem correções e auto-correções ${ }^{(9)}$.

No desenvolvimento normal da linguagem, na faixa etária de zero a 11 meses, podem ser observadas as seguintes características ${ }^{(10)}$ :

- RN e um mês: apresenta choro como reação biológica à dor e à fome, as vocalizações são esporádicas e reflexas, acorda e assusta-se com os sons intensos, acalma-se com a voz da mãe;

- dois e três meses: o choro se torna diferenciado, as vocalizações e risos parecem estar relacionados a sensações de bem estar e apresentam variação quanto à altura e duração, reage a fala humana (sorri, olha, vocaliza), apresenta atenção ao som, procura a fonte sonora;

- quatro e cinco meses: surgem os "jogos vocais" ou "autobalbucio", balbucio indiferenciado com repetição da 
mesma sílaba e olha quando é chamado;

- seis e sete meses: participa com mais frequência e ativamente da interação com os cuidadores através do riso, expressão facial, movimentação corporal e vocalizações, imita certos sons feitos por outras pessoas, balbucio diferenciado com repetição contínua de diferentes sílabas, manifesta seus desejos olhando ou apontando para os objetos, sem dirigir-se ao outro;

- oito e nove meses: surgem os comportamentos comunicativos intencionais, dirige-se ao outro, repete sons emitidos pelos outros, atende a imperativos rotineiros acompanhados de gestos;

- dez e 11 meses: participa da atividade dialógica por meio de jargão, pode apresentar idiossincrasias e pode repetir palavras ditas pelos outros, todavia, a repetição não tem o mesmo padrão fonológico.

Considerando a escassez de instrumentos de avaliação padronizados da linguagem, que possam ser utilizados por profissionais da área da saúde e a grande demanda de pacientes assistidos no sistema público, procedimentos de triagens, no acompanhamento de lactentes de risco, podem constituir uma alternativa válida. Embora o termo triagem possa transmitir a noção de um procedimento rápido e simples, sabe-se que o processo de selecionar, escolher, separar, de acordo com alguns critérios, pressupõe um primeiro passo para o diagnóstico $^{(11)}$.

A triagem do desenvolvimento da linguagem é um meio razoável de avaliar a integridade de vários subsistemas neurais incluindo a audição, o processamento auditivo central, o desenvolvimento cognitivo, a função motora de articulação, de praxia de membros superiores, a visão e o processamento central da informação visual. A dificuldade da criança em adquirir a linguagem nos padrões normais é quase sempre um indício de algum tipo de problema de desenvolvimento subjacente. Entre os instrumentos temos a Escala ELM - Early Language Milestone $^{(12)}$, a Escala Bayley ${ }^{(13)}$ e o Teste de Denver ${ }^{(14)}$, os quais são instrumentos que auxiliam na determinação de padrões de comportamentos linguísticos esperados para cada fase do desenvolvimento infantil.

A presente pesquisa objetivou determinar a frequência da ocorrência de elementos pré-linguísticos em crianças normais durante o primeiro ano de vida, e desta forma contribuir para revisar parâmetros para melhor uso clínico.

\section{MÉTODOS}

A amostra desta pesquisa constituiu-se de crianças nascidas a termo e normais, no Hospital de Clínicas de Porto Alegre, selecionadas aleatoriamente para um estudo de coorte. A seleção deste grupo foi feita entre todos os recém-nascidos presentes na maternidade no dia da coleta de dados. As primeiras 33 crianças cujos pais assinaram o Termo de Consentimento Livre e Esclarecido e que completaram todas as avaliações no primeiro ano foram incluídas no estudo. Desta forma as crianças sempre foram as mesmas desde o período neonatal e todas foram submetidas à avaliação do neurodesenvolvimento e da antropometria.

As informações, de acordo com as variáveis, sorriso social, sons inarticulados, balbucio monossilábico, balbucio polissilábico, primeiras palavras/palavras-frase e jargão (classificação dos elementos pré-linguísticos já utilizadas em trabalhos anteriores) $)^{(8,11-12)}$ foram obtidas através do inquérito (informações dos pais), da observação espontânea do examinador e de estímulos facilitadores.

A avaliação do neurodesenvolvimento bem como a técnica de verificar os elementos pré-linguísticos foram observadas nas primeiras 72 horas de vida, com um, dois, três, quatro, cinco, seis, nove e 12 meses de vida, pelo mesmo examinador ${ }^{(12,14-15)}$. Foi montado um banco de dados no programa SPSS (versão 11.5), no qual foi realizada uma análise descritiva com as frequências encontrada em cada item. Para as estimativas das variáveis principais, foram utilizados os intervalos de confiança de 95\%, calculados pelo Teste Fleiss Quadratic no Programa Epitable (Epi Info 6 v. 6.04). Esta pesquisa foi aprovada pelo Comitê de Ética do Hospital de Clínicas de Porto Alegre, protocolado sob o número 94/106.

\section{RESULTADOS}

Das 33 crianças avaliadas, 17 (51,8\%) eram masculinas e $22(66,7 \%)$ eram brancas. O parto foi vaginal em $26(78,8 \%)$. Todas as crianças foram consideradas neurologicamente normais ao nascimento bem como durante o tempo em que duraram as avaliações.

As famílias das crianças provinham das regiões urbanas da Grande Porto Alegre, sendo que 23 (70\%) residiam em casa própria, cinco (15\%) em casa alugada e cinco (15\%) em casas com outros familiares. Quanto à escolaridade dos pais, $42,5 \%$ tinham o primeiro grau incompleto, $28,7 \%$ completaram o primeiro grau, $20,0 \%$ completaram o segundo grau, $7,5 \%$ tinham nível superior e o pai desconhecido, 1,3\%. A renda familiar variou de dois a 15 salários mínimos, com média de 5,7 salários.

Levando em consideração as variáveis principais desta pesquisa, os resultados das frequências encontradas e seus respectivos intervalos de confiança estão representados na Tabela 1.

Quanto ao gênero, não foram constatadas diferenças significativas nas manifestações pré-linguísticas; meninos e meninas apresentaram um desenvolvimento similar, no entanto com um ligeiro predomínio a favor do gênero feminino, o que é frequentemente esperado. Quanto à avaliação do neurodesenvolvimento, bem como do crescimento, todas as crianças foram consideradas normais até o primeiro ano de vida.

Discutiremos o presente estudo comparativamente com o de outros pesquisadores, conforme apresentado na Tabela 2.

\section{DISCUSSÃO}

A presente pesquisa mostra que o sorriso social aparece em $100 \%$ das crianças no terceiro mês de vida, o que está adiantado em relação ao parâmetro da escala ELM ${ }^{(12)}$ que encontrou $75 \%$ para o mesmo período etário.

No que se refere à produção do balbucio monossilábico, observamos nesta amostra o seu aparecimento aos três e aos seis meses de idade em $100 \%$ das crianças, isto foi mais pre- 
Tabela 1. Frequência das manifestações pré-linguísticas em crianças normais no primeiro ano de vida

\begin{tabular}{|c|c|c|c|c|c|c|c|}
\hline $\begin{array}{l}\text { Idade em } \\
\text { meses }\end{array}$ & $\begin{array}{c}\text { Sons } \\
\text { inarticulados }\end{array}$ & $\begin{array}{c}\text { Sorriso } \\
\text { social }\end{array}$ & $\begin{array}{c}\text { Balbucio } \\
\text { monossilábico }\end{array}$ & $\begin{array}{c}\text { Balbucio } \\
\text { polissilábico }\end{array}$ & $\begin{array}{c}\text { Primeiras } \\
\text { palavras/ } \\
\text { Palavras-frase }\end{array}$ & Jargão & Frases \\
\hline & $\begin{array}{l}n \quad \% \\
\text { [IC 95\%] }\end{array}$ & $\begin{array}{l}n \quad \% \\
{[\text { IC 95\%] }}\end{array}$ & $\begin{array}{l}\mathrm{n} \quad \% \\
\text { [IC 95\%] }\end{array}$ & $\begin{array}{l}\mathrm{n} \quad \% \\
{[\text { IC 95\%] }}\end{array}$ & $\begin{array}{l}\mathrm{n} \% \\
\text { [IC 95\%] }\end{array}$ & $\begin{array}{l}\mathrm{n} \\
{[\text { IC }}\end{array}$ & $\begin{array}{lr}\text { n } & \% \\
\text { [IC } & 95 \%]\end{array}$ \\
\hline 1 & $\begin{array}{cc}33 & 100 \\
{[87} & -100]\end{array}$ & $\begin{array}{cc}4 & 12,1 \\
{[4} & -29]\end{array}$ & & & & & \\
\hline 2 & & $\begin{array}{lr}30 & 90,9 \\
{[74,5} & -90]\end{array}$ & & & & & \\
\hline 3 & & $\begin{array}{lr}33 & 100 \\
{[87} & -100]\end{array}$ & $\begin{array}{cc}2 & 6 \\
{[1} & -21,6]\end{array}$ & & & & \\
\hline 4 & & & $\begin{array}{c}14 \quad 42,4 \\
{[26-61]}\end{array}$ & & & & \\
\hline 5 & & & $\begin{array}{c}32 \\
{[82-100]}\end{array}$ & $\begin{array}{cc}14 & 42,4 \\
{[26} & -61]\end{array}$ & & & \\
\hline 6 & & & $\begin{array}{cr}33 & 100 \\
{[87} & -100]\end{array}$ & $\begin{array}{c}29 \quad 87,8 \\
{[71-96]}\end{array}$ & & & \\
\hline 9 & & & & $\begin{array}{c}33 \quad 100 \\
{[87-100]}\end{array}$ & $\begin{array}{l}17 \quad 51,5 \\
{[34-69]}\end{array}$ & $\begin{array}{cc}1 & 3 \\
{[0,2} & -17,5]\end{array}$ & \\
\hline 12 & & & & & $\begin{array}{c}21 \quad 63,6 \\
{[45-79]}\end{array}$ & $\begin{array}{lr}2 & 6 \\
{[1} & -21,6]\end{array}$ & 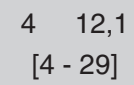 \\
\hline
\end{tabular}

Legenda: [IC 95\%] = Intervalo de Confiança de 95\%.

Tabela 2. Desenvolvimento pré-linguístico: comparação entre estudos

\begin{tabular}{|c|c|c|c|c|c|c|c|c|}
\hline \multirow[t]{2}{*}{ Manifestações pré-linguísticas } & \multicolumn{2}{|c|}{$\begin{array}{c}\text { Coplan, } 1982 \\
n=191\end{array}$} & \multicolumn{2}{|c|}{$\begin{array}{c}\text { Lima, } 1997 \\
\quad n=44\end{array}$} & \multicolumn{2}{|c|}{$\begin{array}{c}\text { Lima, } 2004 \\
n=46\end{array}$} & \multicolumn{2}{|c|}{$\begin{array}{l}\text { Presente estudo } \\
\qquad n=33\end{array}$} \\
\hline & *mês & $(\%)$ & mês & $(\%)$ & mês & $(\%)$ & mês & $(\%)$ \\
\hline Sons inarticulados & 1,5 & $(75)$ & - & & - & & 2,0 & $(100)$ \\
\hline Sorriso social & 3,0 & $(75)$ & - & & - & & 3,0 & $(100)$ \\
\hline Balbucio monossilábico & 8,1 & $(75)$ & 8,0 & $(100)$ & 8,0 & $(75)$ & 6,0 & $(100)$ \\
\hline Balbucio polissilábico & 9,0 & $(75)$ & 9,0 & $(100)$ & 9,0 & $(54)$ & 9,0 & $(100)$ \\
\hline Primeiras palavras/palavras-frase & 11,3 & $(50)$ & 12,0 & $(75)$ & 12,0 & $(38)$ & 12,0 & $(63,6)$ \\
\hline Jargão & 10,9 & $(25)$ & - & & - & & 12,0 & $(6,1)$ \\
\hline
\end{tabular}

Legenda: * idade em meses da aquisição do aspecto linguístico e \% de crianças

coce em relação à faixa etária dos grupos aos quais estamos comparando.

Quanto ao balbucio polissilábico, encontramos 14 (42,4\%) a partir dos cinco meses e $100 \%$ com nove meses, enquanto um dos estudos ${ }^{(11)}$ encontrou 25 crianças $(54,3 \%)$ que produziam sílabas repetidas até os nove meses, uma frequência bastante abaixo do encontrado na presente amostra e também aquém do encontrado em outra pesquisa do mesmo autor ${ }^{(8)}$ na qual a frequência foi igual à nossa, de $100 \%$, aos nove meses. Frequências intermediárias como $75 \%$ para esta mesma idade têm sido descritas ${ }^{(12)}$.

Nesta pesquisa vamos considerar que o uso da primeira palavra verdadeira e palavra frase como sendo a mesma categoria, em função da dificuldade de classificá-las por não ter uma terminologia padronizada nas diversas línguas e também pela superposição temporal em que elas podem ocorrer. As primeiras palavras foram provavelmente produzidas após o sexto mês de vida, uma vez que na revisão dos nove meses $51,5 \%$ das crianças já apresentavam, e até o fim do primeiro ano houve um pequeno aumento de só $12 \%$. Ainda quanto à produção das primeiras palavras houve um ligeiro predomínio do gênero feminino aos 12 meses, das 21 crianças, nove eram meninos e 12 meninas.

As manifestações do uso da linguagem expressiva, como a primeira palavra, o uso de frases com três palavras e o jargão, mostrou diferenças maiores em relação aos percentuais por idade de crianças com estas aquisições entre os estudos comparados, o que está de acordo com a regra do desenvolvimento normal, em que se espera maior variabilidade de aquisição para as funções mais complexas e elaboradas. Deve-se considerar também que neste período os pais têm mais dificuldades em caracterizá-las. 


\section{CONCLUSÃO}

Os resultados da presente pesquisa estabelecem um padrão normal de desenvolvimento das manifestações pré-linguísticas. Porém, apesar de existirem padrões, até mesmo universais, para a aquisição da linguagem, é importante salientar que as variações individuais, em determinados casos, podem ocorrer sem caracterizar patologia. Essas variações, tanto quantitativas como qualitativas, dos componentes da linguagem inicial são relevantes, de fato essenciais, para a compreensão dos mecanismos subjacentes ao desenvolvimento normal da linguagem.
Há necessidade de revisar marcos do desenvolvimento normal em diferentes épocas, em regiões geograficamente distintas, entre tipos raciais, entre diferentes condições sociais, entre outros. Esses dados serão úteis não só para verificar possíveis anormalidades em aplicações de testes de triagem, bem como para levantar possíveis mudanças de padrões de desenvolvimento que ocorram por fatores evolutivos, quer de origem social ou mesmo genética. Os resultados indicam a necessidade de revisar dados de avaliação de linguagem já estabelecidos por pesquisas realizadas no passado que constituem padrões de avaliação em uso atualmente.

\begin{abstract}
Purpose: The aim of this study was to determine the frequency and occurrence of pre-linguistic elements in normally developing infants during their first year of life. Methods: The sample comprised 33 full-term normal children, born at the Hospital de Clínicas de Porto Alegre, randomly selected for a cohort study. The information regarding the variables social smile, inarticulate sounds, monosyllabic babbling, polysyllabic babbling, first words, jargon and sentences, were obtained through an inquiry (parental information), spontaneous observation by the examiner, and facilitating stimuli. Results: These results set a pattern of normal development of pre-linguistic manifestations. Conclusions: The results showed the need to review language assessment data already established by previous studies and evaluations currently applied.
\end{abstract}

Keywords: Language development; Phonetics; Child

\section{REFERÊNCIAS}

1. Sevcik RA. Comprehension: an overlooked component in augmented language development. Disabil Rehabil. 2006;28(3):159-67.

2. Chomsky N. Language and mind. New York: Harcourt, Brace \& World; [1968].

3. Bates E, Marchman V, Thal D, Fenson L, Dale P, Reznick JS, et al. Developmental and stylistic variation in the composition of early vocabulary. J Child Lang. 1994;21(1):85-123.

4. Castaño J. Bases neurobiológicas del lenguaje y sus alteraciones. Rev Neurol. 2003;36(8):781-5.

5. Rescorla L, Mirak J. Normal language acquisition. Semin Pediatr Neurol. 1997;4(2):70-6.

6. Pereira MR, Funayama CAR. Avaliação de alguns aspectos da aquisição e desenvolvimento da linguagem de crianças nascidas pré-termo. Arq Neuropsiquiatr. 2004;62(3A):641-8.

7. Fenson L, Bates E, Dale P, Goodman J, Reznick JS, Thal D. Measuring variability in early child language: don't shoot the messenger. Child Dev. 2000;71(2):323-8.

8. Lima MCMP. Avaliação de fala de lactentes no período pré-linguístico: uma proposta para triagem de problemas auditivos [tese]. Campinas: Faculdade de Ciências Médicas da Universidade Estadual de Campinas; 1997.
9. Pedroso F, Rotta NT. Transtornos da linguagem. In: Rotta NT, Ohlweiler L, Riesgo RS. Transtornos da aprendizagem: abordagem neurobiológica e multidisciplinar. Porto Alegre: Artmed; 2006. p.131-50.

10. Harding CG, Golinkoff RM. The origins of intentional vocalizations in prelinguistic infants. Child Dev. 1979;50(1):33-40.

11. Lima MCMP, Barbarini GC, Gagliardo HGRG, Arnais MAO, Gonçalves VMG. Observação do desenvolvimento de linguagem e funções auditiva e visual em latentes. Rev Saúde Pública $=\mathrm{J}$ Public Health. 2004;38(1):106-12.

12. Coplan J, Gleason JR, Ryan R, Burke MG, Williams ML. Validation of an early language milestone scale in a high-risk population. Pediatrics. 1982;70(5):677-83.

13. Bayley N. Manual for the Bayley scales of infant development. 2nd ed. San Antonio, TX: The Psychological Corporation; 1993.

14. Frankenburg WK, Dodds J, Archer P, Shapiro H, Bresnick B. The Denver II: a major revision and restandardization of the Denver Development Screening Test. Pediatrics. 1992;89(1):91-7.

15. Paine RS. Neurological examination of infants and children. Pediatr Clin North Am. 1960;7:471-510. 